\title{
Improvements in the O\&M modelling of floating offshore wind farms
}

\author{
G. Rinaldi, P.R. Thies \& L. Johanning \\ Renewable Energy group, University of Exeter, Penryn Campus, Treliever Road, TRlo9FE, Penryn, \\ Cornwall, $U K$
}

\begin{abstract}
Operation and maintenance are widely recognized as major areas for cost reduction in offshore wind installation. This paper explores improvements in the O\&M planning capabilities for the floating offshore wind sector. Computational tools have been developed in the last decades to support the strategic planning of onshore and offshore wind O\&M interventions. However, adaptations will be needed in order to capture the novel operational requirements of floating devices with respect to traditional bottom-fixed turbines. In this paper, specific adjustments to offshore O\&M planning computational tools in order to capture floating farms capabilities are identified and implemented. The effects of these amendments on the overall maintenance strategy, as well as related key performance indicators, are tested for the same case study. The comparison between bottom-fixed and floating offshore wind projects allows to quantify the impacts of using floating platforms, and provides indications for future improvements in floating wind O\&M planning.
\end{abstract}

\section{INTRODUCTION}

The offshore wind sector has rapidly progressed in the last decades (GWEC 2018), and has become one of the most cost-effective and most reliable sources of renewable energy. Traditionally, bottomfixed foundations (e.g. monopile) have been employed as support structures for wind turbines in locations with a water depth up to $50 \mathrm{~m}$ (The Carbon Trust 2015). Beyond this limit, the manufacture and installation of fixed foundations becomes impractical and economically unviable (Crown Estate Scotland and ORE Catapult 2018). As such, despite margins for improvement with this technology certainly still exist, solutions exploiting a floating platform, which can be deployed in deeper locations, are increasingly being proposed.

However, as for most nascent technologies, a number of uncertainties exist in relation to their development. Among these, an important one is related to the operation and maintenance (O\&M) of the devices. Since O\&M has been identified in several studies (Dawid et al. 2012; Dinwoodie et al. 2013; Rinaldi et al. 2018; Shafiee and Kolios 2015) as one of the major contributors to the overall costs of offshore wind projects, covering around $25-30 \%$ of these, it is important to investigate how the change from fixed to floating foundations will affect this aspect. An example of O\&M modelling of floating devices is presented in (Gray 2019), in which modelling efforts focus in capturing the failures of two different technologies (wind and wave energy converter) mounted in the same hybrid device.

In this context, the aim of this paper is twofold. Firstly, the specific adjustments implemented to simulate the operation of floating wind devices are presented and discussed. Secondly, these adjustments are exploited to make a comparison between bottom-fixed offshore wind (BFOW) and floating offshore wind (FLOW), and to verify to what extent operating and maintaining floating foundations instead of fixed ones affects the key performance indicators (KPIs) of an offshore farm.

A case study considering two analogous offshore wind farms, one fixed and the other floating, is used for this purpose. The changes required to capture the O\&M dynamics of the two kinds of devices are implemented, and the KPIs for the two projects compared. The aim is to understand the reasons for eventual differences, and elaborate on the support that O\&M planning can provide in the transition towards a cost-effective FLOW sector.

In order to achieve this task, a simulation model for the characterization of the key performance indicators of an offshore energy farm, with particular emphasis towards the O\&M strategy planning, is used. This tool was developed by the authors, and has been applied for other O\&M applications in the bottom-fixed offshore wind, wave and tidal energy sectors (see Rinaldi et al. 2016; Rinaldi et al. 2017; Rinaldi et al. 2017; Rinaldi et al. 2019). The tool has also been verified for its intended use (Rinaldi et al. 
2018). Several tools of this kind have been developed in recent years to provide support in the offshore renewable energy (ORE) sector (Hofmann 2011; Seyr 2016; Shafiee and Sørensen 2019), but new functionalities will be required for FLOW technologies.

Thus, the rest of this paper is organized as follows. In section 2, the methodology adopted to build the case study, model FLOW farms and interpret the KPIs estimated by means of computational simulation is described. In section 3 , the outcomes of the simulations, selected among those relevant for a comparison between technologies, are presented and explained. Section 4 discusses the results of this comparative assessment in view of the wider implications for the O\&M planning and overall viability of FLOW farms. Finally, in the last section, conclusions are drawn together with suggestions for future work.

\section{METHODOLOGY}

This section provides an overview of the instruments needed to assemble a comparative case study between BFOW and FLOW technologies, providing the following details:

- The computational tool used for the estimation of the KPIs, together with the features added in order to capture FLOW O\&M;

- The dataset describing the reference case study, a BFOW farm modelled in previous work (Rinaldi et al. 2019); and

- The modifications to the reference case study made in order to simulate a FLOW farm.

\subsection{Computational tool and adjustments for FLOWs}

The O\&M modelling tool used to evaluate the performance of the different ORE farms was originally conceived to provide support to ocean renewables owners or operators in their planning tasks. In fact, the tool aids the decision-making process needed for the effective assets management of an ORE farm. It can be used at either the beginning of the project or when the farm is already operational, and provides support for the long-term ("strategic") decisions, i.e. for 5-15 years of operation or even the whole project lifecycle. This model is based on a common and established methodology, the Markov Chain Monte Carlo (MCMC) approach, which combines random processes representing a sequence of events (for example from a functioning state to failure) with repeated sampling of the same scenario subject to random variations. In this way, for a sufficient number of simulations, the averaged results approximate the actual representation of the system. A statistical approach of this kind permits to obtain confidence intervals on the estimates, which in turn addresses the underlying uncertainties in the system.

A series of input data is needed to allow the model to recreate a simulation that is satisfactorily representative of the real ORE farm. These include, but are not limited to:

- metocean data of the offshore location where the devices will be deployed;

- information on the taxonomy (subassemblies and components), reliability (failure rates, redundancies, criticalities, dependencies) and power performance of the devices;

- specifics of the access systems (vessels, workboats, helicopters) that will be used for the maintenance of the farm; and

- the planned maintenance schedule.

Once the initial input data are gathered, a series of estimates describing the farm's performance are generated in terms of energy yield, reliability, availability and financial indicators, e.g. revenue and O\&M costs. Thus, eventual bottlenecks or underlying issues can be identified, providing useful insights for the decision-makers and allowing them to propose suitable mitigation measures.

A simplified workflow diagram of the computational model used in this study is shown in Figure 1 (Rinaldi et al. 2016). More details on the mechanisms and constraints regulating its functioning are discussed in Rinaldi et al. 2017.

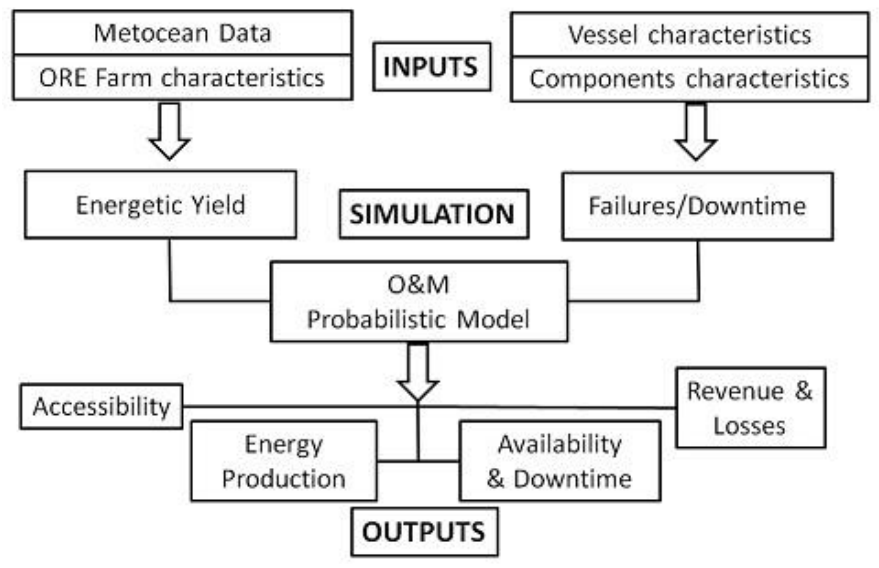

Figure 1. Simplified workflow Diagram of the O\&M model (Rinaldi et al. 2016).

The model was originally developed for the marine energy sector, allowing for flexibility across a range of different technologies (offshore wind turbines and wave and tidal energy converter). However, a series of adjustments is needed in order to make the tool suitable to capture the dynamics of FLOW farms.

One of the main advantages of floating devices is that they can be assembled in port before being towed to the offshore location, taking advantage of a sheltered site as well as of the port infrastructure. For the same reasons, floating devices can also be 
maintained in port, especially during major interventions, avoiding the expenses related to the charter of big maintenance vessels and bypassing the limitations due to poor weather. Therefore, in order to make the model able to take into account these mechanisms, the possibility to simulate on-shore (in port) maintenance, for one or more specific components of the device, has been added as a functionality of the tool.

In addition, the possibility to specify whether the repair or replacement task has to be concluded in one intervention or can be separated in multiple shifts, has been included. In reality, it can be expected that, once towed onshore, a device will remain at port for some time in order to take advantage of the operation to perform multiple interventions. However, two modelling possibilities are implemented in the O\&M tool to provide major flexibility during simulations. In the first case, it is assumed that the access system (e.g. a tugboat) goes to the offshore location, tows the device to port, awaits for maintenance, brings the device back to the offshore site and returns to port. In the second case the same steps can happen in separate stages, with a first stage considering the transit from port to the device and return, then maintenance onshore, and finally a third stage to bring the device back to its offshore site and allow the vessel to return to port. These two options are graphically represented in Figure 2.

The difference is that in the first case suitable weather conditions (i.e. a "weather window") must be available for the duration of the whole operation, whereas in the second case the weather related restrictions apply only to the stages involving actual navigation but not the work in port. In other words, a long weather window is needed to let the operation begin in the first case, while two shorter and independent weather windows are needed in the second case. The first option ("continuous tow-to-port maintenance") represents an intervention that has to be completed in one go, which might be preferable when the onshore maintenance is rapid (e.g. repair of a minor component), or during the summer months, when suitable and continuous weather conditions are more likely to happen. Or again, when a vessel is chartered for short periods or one type of vessel (not necessarily the same one) is continuously going back and forth from port to tow devices in/out. On the contrary, the second option ("discontinuous tow-toport maintenance") might be preferable when the vessel is owned or can be used for other tasks while a component is repaired (opportunistic maintenance), or when the maintenance intervention onshore lasts for a long time, or when the probability of having long and continuous weather windows is low, e.g. winter months.

Disconnection and reconnection of the floating platform, representing the activities needed to detach the device from its mooring system and power cable, and then restore it, for tow-to-port operations, are also considered. The duration of these events is added to the total duration of an intervention for those components requiring onshore maintenance.

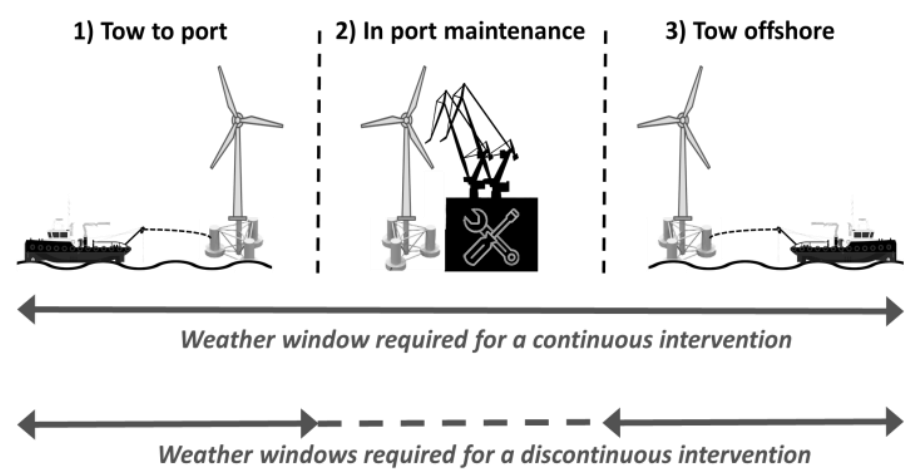

Figure 2. Graphical representation of the difference between continuous and discontinuous onshore (in port) maintenance in terms of the required weather window(s).

\subsection{Reference case study - BFOW}

The first simulated scenario explores an example BFOW farm inspired to an existing one, the Westermost Rough Wind Farm (Westermost Rough Wind Farm 2017), located off the east coast of the United Kingdom. The O\&M port for maintenance of this farm is the Royal Dock Grimsby, located approximately $40 \mathrm{~km}$ from the installation site. In this study, the farm is assumed as 25 offshore wind turbines rated at $8 \mathrm{MW}$ each. The specifications of this case study, including metocean data, maintenance fleet and device properties, have been extracted from previous work (Rinaldi et al. 2019). Metocean data (hindcast wave and wind time-series) cover a 10 years period (1990 - 1999) with a resolution (timestep) of three hours. Three kinds of access systems, namely crew transfer vessel (CTV), field support vessel (FSV) and heavy-lift vessel (HLV) are used for maintenance of the wind turbines. Limitations are applied to the maximum wind speed and wave height the vessels can operate in. Since the optimization of the maintenance fleet is beyond the scope of this work, it has been decided to remove any limitations on the number of vessels available, meaning that there are as many as needed for any kind of maintenance intervention. Finally, the parameters defining the subsystems and components of the wind turbines, together with failure data and replacement costs, are based from (Carroll et al. 2016) and adjusted as described in (Rinaldi et al. 2019).

\subsection{Comparative FLOW cases - Assumptions}

In order to conduct a fair comparison, the same input data set has been used for the two cases, i.e. the BFOW and the FLOW farms. However, a num- 
ber of adjustments has been applied in order to make the two scenarios suitable for the purposes of this investigation. The following variations and assumptions, devised according to previous literature and in order to create a suitable comparison, have been applied to the FLOW farm:

- The two offshore farms are situated in the same location, and therefore affected by the same environmental conditions;

- The devices used in the two hypothetical farms are the same, therefore having the same kind and number of components (taxonomy) as well as reliability and maintainability characteristics (e.g. failure rates and replacement costs). This last concept applies also to the support structures, i.e. despite being one floating and the other fixed, the same values of reliability and maintainability are used for both technologies;

- The transit time for a maintenance vessel towing the device (either from the offshore location to port or vice versa) is $30 \%$ higher than the transit time without device (i.e. it is assumed that a vessel towing a device will be slower than without);

- The metocean limits that constrain the vessels for tow-to-port operations (i.e. to bring the device to the onshore port for maintenance) are $70 \%$ lower than the ordinary wave height, wind speed and current speed limits for the same vessels;

- For the FLOW farm scenario, it is established that the device is towed to port for maintenance on 8 (out of 16) components. These are the components usually requiring major interventions, e.g. hub, generator, converter, yaw system, etc. However, the typology of vessel associated to every specific component remains the same;

- The FLOW farm case is divided in two subscenarios. In the first, the onshore maintenance has to be continuous, i.e. completed in one intervention as described in section 2.1. In the second, maintenance intervention can be discontinuous, i.e. weather restrictions apply only to the individual towing stages;

- Disconnection and reconnection times are assumed to last both for one hour, i.e. a total of two hours is added to onshore maintenance activities.

\section{RESULTS}

Once the different cases are set up, the O\&M model is used to estimate the KPIs depicting the three scenarios (BFOW, FLOW - continuous and FLOW - discontinuous). A selection of the energetic and financial indicators considered most relevant for the comparison is provided in Table 1 . In this table, the performance of the simulated farms is compared for the following indicators:

- electricity generated and lost due to unexpected failures, for the entire farm ( 25 devices) over the whole simulated period (10 years);

- energy-based availability, capacity factor and equivalent hours;

- total revenue generated from the sale of electricity;

- direct O\&M costs (sum of expenses due to repair or replacement of the components, salary of technicians and vessels use); and

- the final generated income.

A strike price of $£ 57.5 / \mathrm{MWh}$, in line with the CfD auction for offshore wind farms scheduled for commissioning in 2022/2023 (CfD Round Two results 2020), has been assumed to calculate the revenue generated by the sale of electricity.

Table 1. Comparison of the results obtained for the three simulated scenarios.

\begin{tabular}{llll}
\hline Quantity & BFOW & FLOW $_{1 C^{*}}$ & FLOW $_{2 D^{*}}$ \\
\hline $\begin{array}{l}\text { Energy delivered } \\
\text { [GWh] }\end{array}$ & 7642.2 & 7614.9 & 7669.1 \\
$\begin{array}{l}\text { Energy lost } \\
\text { [GWh] }\end{array}$ & 254.8 & 282.1 & 227.9 \\
$\begin{array}{l}\text { Availability [\%] } \\
\text { Capacity factor }\end{array}$ & 96.7 & 96.4 & 97.1 \\
[\%] & 43.6 & 43.4 & 43.7 \\
$\begin{array}{l}\text { Equivalent hours } \\
\text { Total revenue }\end{array}$ & 3821.1 & 3807.4 & 3834.5 \\
[m£] & 439.4 & 437.8 & 440.9 \\
$\begin{array}{l}\text { O\&M costs [m£] } \\
\text { Total income }\end{array}$ & 46.6 & 54.4 & 56.4 \\
[m£] & 392.7 & 383.3 & 384.5 \\
\hline
\end{tabular}

$* 1 \mathrm{C}=$ onshore continuous maintenance, $2 \mathrm{D}=$ onshore discontinuous maintenance.

The results show that for the FLOW cases the choice between continuous and discontinuous interventions for those components requiring onshore maintenance does make a difference. In fact, when compared against the BFOW case, the KPIs are lower for the case with continuous onshore operations $\left(\mathrm{FLOW}_{1 \mathrm{C}}\right)$ and higher for the case with continuous onshore operations $\left(\mathrm{FLOW}_{2 \mathrm{D}}\right)$. However, the O\&M costs are lowest for the BFOW case, followed by the $\mathrm{FLOW}_{1 \mathrm{C}}$ and the $\mathrm{FLOW}_{2 \mathrm{D}}$ last. As a result, when O\&M costs are taken into account, the ranking changes again, with the BFOW generating the highest income followed by the $\mathrm{FLOW}_{2 \mathrm{D}}$ and the FLOW $_{1 \mathrm{C}}$ last.

In order to explain these results, it must be considered that while the energy production is similar for the three scenarios, with relatively small differences of $\pm 0.4 \%$, the O\&M costs for the two FLOW scenarios are $+17 \%$ and $+21 \%$ higher than the BFOW respectively. The same reasoning applies 
between the two FLOW cases, with the FLOW 2 D farm generating $0.7 \%$ more electricity than the FLOW $_{1 \mathrm{C}}$, but also causing $3.7 \%$ higher O\&M costs.

In terms of reliability and maintainability of the components, the results are similar for the three cases, with 517, 515 and 519 simulated maintenance tasks for the BFOW, FLOW $1 \mathrm{C}$ and FLOW 2 D scenarios respectively. These are all corrective interventions, since planned maintenance was not included for the sake of simplicity. The small differences are motivated by the different timings required to maintain a certain component under the different assumptions made for each scenario, as well as the related weather windows availability. In other words, the quicker the maintenance interventions are (either because a suitable weather window opens earlier or the overall maintenance time is lower), the higher will be the energy production, but the same components will also get more time (opportunity) to fail. The investigation of the components' contribution to the system reliability goes beyond the scope of this paper. Nonetheless, the distribution of failures among the considered components, for the three scenarios, is shown in Figure 3. From this histogram, it can be seen how the simulated failures do not vary significantly across the three scenarios, which had to be expected since the components data used in all three cases are the same. Besides, the contribution of each component to the total number of failures is consistent with the individual failure rates used for the investigation (Rinaldi et al. 2019).

\section{DISCUSSION}

The results obtained in this comparative work highlight once more the importance of improving the O\&M strategy and the strategic asset management for ORE farms. In fact, just a small variation in the maintenance policy of some of the components (from continuous to discontinuous in port maintenance) can make a significant difference in terms of the generated income. In the example provided in this study, this is $\sim £ 9.5 \mathrm{~m}$ over 10 years (difference between the best case, BFOW, and the worst, FLOW $_{1 \mathrm{C}}$ ), and this outcome is in relation to a small/medium farm at a relatively short distance from the O\&M port. More significant differences would certainly be obtained for cases involving multiple choices on the assets, more devices to maintain, at a longer distance from shore and for a longer period.

In this regard, it should be noticed that this investigation, like all other O\&M planning studies, is based on a number of assumptions. These have been described in detail, and do not affect the validity of the comparison, but certainly affect the results obtained. Hence, further simulations are required to explore the effects of different O\&M strategies for

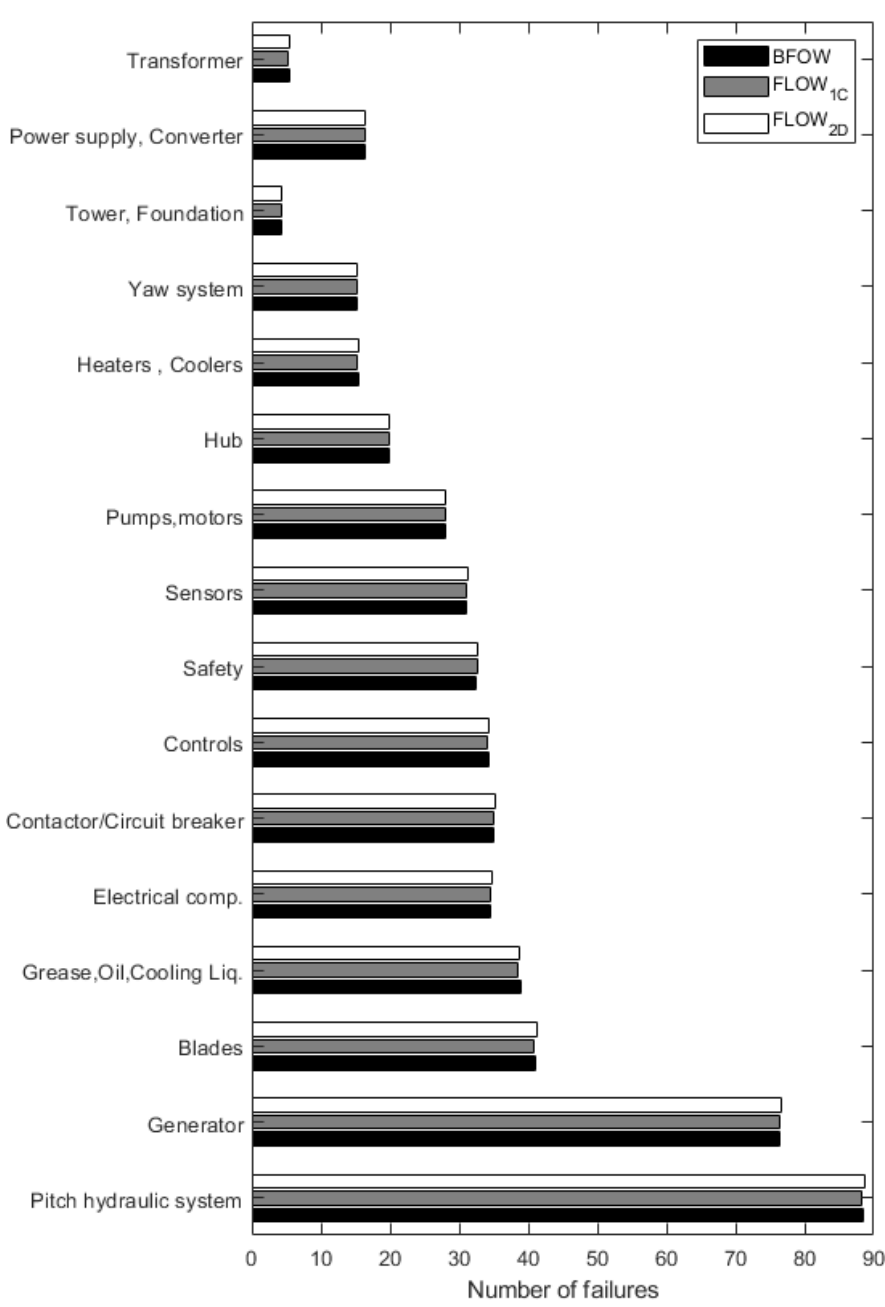

Figure 3. Number of failures per component during the simulated period. Comparison among the three simulated scenarios

FLOW farms or assess the potential impact of each parameter on the KPIs. For instance, in the second variation $\left(\mathrm{FLOW}_{2 \mathrm{D}}\right)$, the time needed to disconnect and reconnect the devices for major maintenance operations was fixed to one hour. A sensitivity analysis may be implemented in order to assess the effects of increasing or reducing this duration on the farm's availability and maintainability and would be able to inform technological requirements regarding the connections.

Similarly, the fact of considering the same failure data for both kinds of support structures (monopile and floating platform) is a big but necessary simplification due to the lack of suitable references with floating platforms. However, this assumption does not cause a noteworthy difference in the results because of the very low failure rate, and therefore related impact on maintenance. Besides, this is a reasonable assumption considering that support structures are over engineered with safety factors, and therefore rarely require major structural interventions.

It is also acknowledged that modelling the same wind turbine might lead to an oversimplification of the problem because turbines for floating platform 
will probably be adjusted in order to satisfy the additional requirements due to the instability of the foundation and tilting angles.

The same consideration is valid for the variation in metocean limits of the vessels when onshore maintenance is required, i.e. the device has to be towed to port. In this case, it was only assumed that stricter limits would apply if a device is being towed, but other factors like a variation in transit speed might also play a role. Again, a sensitivity analysis with respect to maintenance assets capabilities will be useful to derive further insights into the maintenance of floating devices.

\section{CONCLUSIONS}

In this work, the upcoming transition from bottom-fixed to floating foundations in the offshore wind sector is addressed from the point of view of the O\&M strategic planning. In particular, this change in technology is investigated in terms of the adjustments that will be needed in the computational tools generally used to improve the reliability, availability, maintainability and profitability of a project. The main adjustment is identified in the capability to model one of the characteristic features of floating devices: the possibility to be maintained onshore where port infrastructure facilitate major interventions. As such, the O\&M simulation tool is improved with the capability to model this aspect and discern between two approaches: continuous and discontinuous operations.

This feature provides higher flexibility in modelling the maintenance of each component, and can be combined with other pre-existing capabilities in order to offer more choices to the decision-maker. For example, the seasonality restrictions on maintenance assets, which are generally used to model a vessel chartered only during specific periods, can be combined with a discontinuous onshore maintenance. Vice versa, continuous onshore maintenance can be chosen in combination with maintenance assets that are property of the farm or with opportunistic maintenance, i.e. perform other tasks while the device is being repaired.

A case study is implemented in order to understand the implications of choosing one maintenance strategy over the other, and having a BFOW farm as a reference. According to this, the choice on maintenance strategy can have an impact on both the productivity KPIs and the direct O\&M costs, and the variations in these aspects must be balanced in order to verify what the most cost-effective solution is. The influence of the specific input dataset chosen for the case study is discussed, together with the possible consequences of choosing different scenarios.

Nevertheless, further work is needed in order to extend the outcomes of this study and fully under- stand the novelty of FLOW farms from an O\&M planning perspective. This will include the simulation of more cases with more informed datasets, possibly derived from existing and planned projects, and including sensitivity analysis on important parameters such as the number of devices or the distance from the shore. Similarly, the cost analysis can be further improved by providing ways of measuring the impact of the individual contributors to the overall operation, e.g. the costs for disconnecting mooring lines and cables, for towing, for onshore maintenance work and then the reverse operation for reinstalling the floating wind turbine on site. In addition, despite this work is mainly focused on the O\&M characterization and improvement, capital expenses and their contribution to the overall costs might be included in order to obtain holistic indications on the improvement of FLOW projects viability.

In conclusion, comprehensive computational modelling allows to inform and support decisionmaking for the strategic O\&M planning of floating offshore wind turbines. The computational tools are available and adaptable to incorporate the requirements of future FLOW farms.

\section{ACKNOWLEDGMENTS}

This project has received funding from the European Union's Horizon 2020 research and innovation programme under the project "FLOTANT", grant agreement number 815289 .

\section{REFERENCES}

Carroll, James, Alasdair McDonald, and David McMillan. 2016. "Failure Rate, Repair Time and Unscheduled O\&amp;M Cost Analysis of Offshore Wind Turbines." Wind Energy 19(6): 1107-19.

http://doi.wiley.com/10.1002/we.1887 21, 2017).

"CfD Round Two Results." 2020. https://www.4coffshore.com/news/cfd-roundtwo-results-are-in2c-offshore-wind-cheaperthan-gas-and-nuclear-nid6373.html (March 28, 2020).

Crown Estate Scotland, and ORE Catapult. 2018. Macroeconomic Benefits of Floating Offshore Wind in the UK. https://www.crownestatescotland.com/mapsand-publications/download/219.

Dawid, Rafael, David Mcmillan, and Matthew Revie. 2012. "Review of Markov Models for Maintenance Optimization in the Context of Offshore Wind." : 1-11.

Dinwoodie, Iain et al. 2013. "Development of a Combined Operational and Strategic Decision Support Model for Offshore Wind." 00: 24-25.

Gray, Anthony. 2019. "Operations and Maintenance Modelling of Floating Hybrid Systems." In 
Wind Europe,

GWEC. 2018. Global Wind Report. www.gwec.net.

Hofmann, Matthias. 2011. "A Review of Decision Support Models for Offshore Wind Farms with an Emphasis on Operation and Maintenance Strategies." Wind Engineering 35(1): 1-16. http://journals.sagepub.com/doi/abs/10.1260/03 09-524X.35.1.1.

Rinaldi, G., P.R. Thies, R. Walker, and L. Johanning. 2017. "A Decision Support Model to Optimise the Operation and Maintenance Strategies of an Offshore Renewable Energy Farm." Ocean Engineering 145: 250-62. http://linkinghub.elsevier.com/retrieve/pii/S002 9801817304717.

Rinaldi, G, P R Thies, and L Johanning. 2017. "A Coupled Monte Carlo - Evolutionary Algorithm Approach to Optimise Offshore Renewables $\mathrm{O}$ \& M." In 12th European Wave and Tidal Energy Conference, Cork, 1-7.

Rinaldi, G, P R Thies, L Johanning, and R T Walker. 2016. "A Computational Tool for the ProActive Management of Offshore Farms." In 2nd International Conference on Offshore Renewable Energy, Glasgow, UK: ASRANet Ltd, 111-15.

Rinaldi, Giovanni, Ajit C. Pillai, Philipp Rudolf Thies, and Lars Johanning. 2018. "Verification and Benchmarking Methodology for O\&M Planning and Optimization Tools in the Offshore Renewable Energy Sector." In Submitted to International Conference on Ocean, Offshore and Arctic Engineering (OMAE), Madrid.

Rinaldi, Giovanni, Ajit C Pillai, Philipp R Thies, and Lars Johanning. 2019. "Multi-Objective Optimization of the Operation and Maintenance Assets of an Offshore Wind Farm Using Genetic Algorithms." Wind Engineering.

Seyr, Helene. 2016. "Operation and Maintenance Models for Offshore Wind Farms Mathematical Structure and Techniques OPERATION AND MAINTENANCE MODELS FOR OFFSHORE WIND FARMS ." (May).

Shafiee, Mahmood, and Athanasios Kolios. 2015. "A Multi-Criteria Decision Model to Mitigate the Operational Risks of Offshore Wind Infrastructures." In Safety and Reliability: Methodology and Applications, , 539-47.

Shafiee, Mahmood, and John Dalsgaard Sørensen. 2019. "Maintenance Optimization and Inspection Planning of Wind Energy Assets: Models, Methods and Strategies." Reliability Engineering and System Safety 192(November 2017).

The Carbon Trust. 2015. Floating Offshore Wind: Market and Technology Review.

"Westermost Rough Wind Farm." 2017. https://orsted.co.uk/en/Generating energy/Offshore wind/Our wind farms (December 12, 2017). 